\title{
Algunos aspectos de la historia social del sida en los inicios de la epidemia
}

\section{Gunther Balarezo López}

Universidad Científica del Sur

\section{RESUMEN}

Se hizo una revisión bibliográfica sobre la historia social de la epidemia del sida, y se analizó y resumió los aspectos más relevantes de diversas publicaciones. El sida fue un problema de salud que tenía un fuerte impacto social por la desinformación, la discriminación, la economía familiar y del país, y la reacción de familiares y amigos ante el diagnóstico positivo, lo cual hizo que esta enfermedad se convirtiera tanto en un problema social como de salud pública. A pesar de ello, los científicos sociales no eran tomados en cuenta para hacer frente a la epidemia, porque la prioridad era conseguir una vacuna que pudiera curar a los infectados con el virus VIH.

\section{PALABRAS CLAVE}

Sida, salud pública, diagnóstico, economía, educación.

\section{ABSTRACT}

There was a literature review on the social history of AIDS epidemic, analyzing and summarizing the most relevant aspects of various publications. AIDS was a health problem that had a strong social impact by misinformation, discrimination, family economy and the country and the reaction of family and friends to a positive diagnosis, which made this disease became a problem both social and public health. However, social scientists were not taken into account to address the epidemic, because the priority was to get a vaccine that could cure those infected with the HIV virus.

1 Sociólogo y magíster en Salud Pública. Docente de pregrado de Medicina en la Universidad Científica del Sur y la Universidad Peruana de Ciencias Aplicadas, y de posgrado de Medicina en la Universidad Ricardo Palma. 


\section{KEY WORDS}

AIDS, public health, diagnosis, economics, education.

\section{Introducción}

El sida se convirtió en una epidemia que pudo haberse expandido rápidamente si no se tomaban las medidas preventivas oportunas, y representó para muchos países una seria amenaza para la salud. Esta enfermedad fue una preocupación para los profesionales de la salud y los científicos sociales, debido a que las características sanitarias y culturales del sida pusieron en evidencia muchas de las limitaciones de la ciencia, de la salud pública y del conjunto de la sociedad. Como consecuencia de ello, las autoridades médicas tuvieron que aceptar, dado que no existía una cura, que el control de la enfermedad dependía de lo que pudieran lograr las ciencias sociales; su rol era el de ayudar en la promoción de cambios en el comportamiento que redujeran efectivamente la tasa de expansión de la infección. Pero el sida era un tema complejo, no solamente porque era una enfermedad nueva, no del todo conocida, que no se podía curar y que causaba la muerte, sino porque además conllevaba cargas afectivas, morales y éticas que la evidenciaban como un problema de orden público ante la sociedad civil².

Históricamente, se descubrió que el virus VIH infectó a las primeras personas durante la década de 1940 y a principios de la de 1950 en África. Un hombre llegó a morir en 1959 como consecuencia de la enfermedad. Posteriormente, los datos sobre el sida datan de finales de los años 60, los cuales se refieren a los miembros de una familia noruega ${ }^{3}$. En 1981, el doctor Michael Gottieb, en la ciudad de Los Ángeles (Estados Unidos), describió, diagnósticó y reportó el primer caso de sida (un tipo muy raro de neumonía en un joven paciente homosexual), y desde entonces los científicos, médicos, biólogos, científicos sociales y la población en general tuvieron muchas dudas acerca de esta enfermedad. El aumento geométrico de los casos de VIH y sida motivaron que esta epidemia fuese de consideración. Pero había otra epidemia menos perceptible pero igualmente peligrosa: la del pánico, creada por una información y prevención teñidas por tabúes y manipulaciones ideológicas ${ }^{4}$.

Asimismo, la aparición del sida provocó muchas reacciones entre el personal de salud y la población en general. Hubo un exceso de confianza

2 Departament de Sanitat i Seguretat Social 1993, Zalduondo y Bernard 1995, Mercer 1985, The Johns Hopkins University 1987, Paicheler 1992, Treicheler 1992, y Aggleton y otros 1994. 3 Family Health International 1998.

4 Child s. f., Meneghin, Moura y Lee 1995, Rubenson 1987, Liskin y otros 1989, Izazola Licea 1996, y Rubenson 1996. 
pensando que el sida no podía afectar a las personas directamente, porque se trataba de una enfermedad de determinados grupos sociales, lo cual fue dar la espalda a la realidad. Por otro lado, se exageró el carácter contagioso del sida y la adopción de una actitud restrictiva fue perjudicial para todos, ya que se limitó las posibilidades que la vida ofrecía ${ }^{5}$.

Al principio de la epidemia, cuando se describió el VIH como el agente causal del sida, se produjeron falsas expectativas acerca de un pronto descubrimiento de una vacuna y se pronosticó, erróneamente, el fin de la epidemia. Estas falsas esperanzas produjeron que grupos poblacionales en los que se había logrado un cambio de conducta significativo en la adopción de sexo seguro pronto olvidaran dichas prácticas, para dar lugar a un nuevo brote epidémico ${ }^{13}$.

\section{Aspectos de salud pública}

El sida era una sigla controvertida de uso habitual. Era una palabra relativamente nueva, con poco más de diez años de historia y sin duda con muchas connotaciones a su alrededor, como miedo, confusión, impotencia, ignorancia, incomprensión, intolerancia, omisión, angustia, dinero, oportunismo, esperanza y solidaridad. Era difícil mencionar en aquellos días una palabra como esa, que tuviera tantos significados y que provocara las más variadas emociones y reacciones en los individuos y en las sociedades ${ }^{6}$.

Según Reyes ${ }^{7}$, en los países latinoamericanos históricamente se han presentado cuatro tipos de actitudes frente a los males, sean estos epidémicos, sociales o económicos: 1) mientras no me afecte, no me preocupo; 2) eso le toca resolverlo a otros, no a mí; 3) que paguen los demás, no yo; y 4) hay que aparentar antes que enfrentar. Como filosofía individual, esta forma de ver las cosas era sumamente dañina, pero lo era infinitamente más si esa era la manera en que los gobiernos y dirigentes latinoamericanos planteaban las soluciones a los problemas.

En el caso del sida, como ha sucedido en otros periodos históricos, las enfermedades de transmisión sexual (ETS) han sido siempre un campo fértil para la interpretación y asignación de significados morales. Tradicionalmente, este tipo de enfermedades eran calificadas como indicadores de conductas sexuales reprobables y clandestinas, y el sida no escapaba a esta condición; aún más, en él se agravaba y se acentuaba ${ }^{8}$.

5 Departament de Sanitat i Seguretat Social 1993.

6 Parker 1993.

7 Reyes 1993.

8 Rodríguez otros 1992. 
Para Frankerberg ${ }^{9}$ y Connor $^{10}$, el sida era considerado como la enfermedad de las «tres epidemias» que se daban al mismo tiempo y que eran: 1) la infección del $\mathrm{VIH} ; 2$ ) el desarrollo del sida y enfermedades oportunistas; y 3) las dificultades y reacciones sociales, económicas y culturales como consecuencia del sida. Por ello, esta enfermedad no podía ser analizada solo desde el punto de vista biomédico, ya que se debía tener en cuenta las implicaciones económicas, políticas, sociales, culturales, geográficas y educacionales, porque era una epidemia que, para reducir su creciente expansión, demandaba un fuerte compromiso político y los recursos necesarios para su prevención ${ }^{11}$. Lo que se llamó la epidemia del sida era, en realidad, el conjunto de las siguientes epidemias: del caos, de los infectados y de los muertos, porque no solo afectaba al paciente, familiares y amigos, sino que demostró tener efectos significativos sobre toda la sociedad; por ello, se utilizaba el eslogan «el sida es un problema de todos» ${ }^{12}$.

Según Cáceres, esta enfermedad se podía resumir en tres grandes etapas, que eran: 1) asociarla con la creencia de que el sida era fundamentalmente una enfermedad de homosexuales; 2) la enfermedad era asociada con otros grupos humanos llamados grupos de alto riesgo; y 3) el número creciente de heterosexuales afectados promovieron una parcial heterosexualización de la enfermedad en la mentalidad de la sociedad, apoyando un cambio de énfasis previo de «grupo de alto riesgo» a «conductas de alto riesgo» ${ }^{13}$.

La caracterización inicial del sida en cuanto a una enfermedad contagiosa, incurable, mortal y ligada principalmente a la homosexualidad hizo asociar la enfermedad con varios estigmas y preconceptos venidos de posiciones referente a la muerte, la contaminación y la sexualidad ${ }^{14}$. Al aparecer el sida en Estados Unidos, se hablaba de "grupos de riesgo» con las cuatro $\mathrm{H}$ : homosexuales, haitianos, hemofílicos y heroinómanos; eran los cuatro jinetes del nuevo Apocalipsis ${ }^{15}$. Las personas aprendieron gradualmente que lo que fue etiquetado como una "plaga gay» (homosexual) o «enfermedad inmunodeficiente de los homosexuales» (GRID, por sus siglas en inglés) no podía ser limitado tan solo a un grupo ${ }^{16}$. Para

\footnotetext{
9 Frankerberg 1998.

10 Connor 1998.

11 Koop 1987, Brandt 1986, y Bastos y otros 1994.

12 Koop 1987, Bronfman y Magis 1996, Fermina Acuba 1993, y Organización Panamericana de la Salud 1993.

13 Cáceres 1995.

14 Brandt 1986, Bastos y otros 1994, y Aller Atucha 1988.

15Cáceres 1995, Frasca 1993, y Del Río y Liguori 1995.

16Cáceres 1995, y Del Río y Liguori 1995.
} 
Del Río y Liguori ${ }^{17}$, el problema fue la caracterización inicial de los llamados grupos de riesgo en Estados Unidos y los países de Europa Occidental, lo cual hizo que la gran mayoría se sintiera «segura» y creyera que estaba a salvo de contraer la infección.

Tener la enfermedad era una sentencia de muerte señalada y anunciada, ver el destino bloqueado, la vida en suspenso y los planes a largo plazo cancelados; estos eran algunos de los efectos emocionales que la definición de sida como enfermedad inevitablemente mortal causó en las personas que vivían con el VIH y el sida. Se supo que esa caracterización fue hecha sobre la base de un análisis de casos avanzados de la enfermedad, y que la verdad era que no se conocía lo suficiente sobre los mecanismos de infección primaria provocada por el VIH y el desorden inmunológico para afirmar que todos los casos seropositivos adquirían sida o que tuvieran un final «invariablemente fatal»; esto dependía en parte del modo de vida de la persona, de cómo estaba su sistema de defensa y de la manera en que el paciente cuidaba de su salud. Toda una línea de activistas con sida se generó precisamente para contestar la ecuación sida $=$ muerte, proponiendo en su lugar los eslóganes silencio = muerte y acción $=v_{i d a}{ }^{18}$. Los activistas del sida consideraban a esta enfermedad como «un desastre natural», empeorada aún más por el prejuicio, la discriminación y una desidia nada benigna ${ }^{30}$.

Geográficamente, en los comienzos de la enfermedad existían diferencias entre las personas infectadas. En el África, el crecimiento de la epidemia era lento y afectó más a quienes sufrían de hambre, malnutrición, pobreza, falta de higiene, equipo sanitario, vicisitudes en el clima y catástrofes naturales; de ahí pasó a las ciudades con el comercio del sexo, mediante la variedad y formas de contactos sexuales y la poligamia. En los países desarrollados (Estados Unidos, Canadá y Europa Occidental), afectó más a los jóvenes urbanos, de clase media para arriba y homosexuales y bisexuales varones; después se propagó a usuarios de drogas por vía intravenosas, prostitutas, minorías, pobres, criminales y a madres e hijos ${ }^{19}$. En el África Subsahariana y en los países tropicales y del Caribe, la transmisión del VIH se realizaba principalmente por la vía heterosexual, seguida de la transmisión perinatal y parenteral; mientras que en los países desarrollados (América del Norte, Europa Occidental, Australia y Nueva Zelanda) la transmisión estaba asociada particularmente con la conducta

17 Del Río y Liguori 1995.

18 Rubenson 1996, Brandt 1986, y Bronfman y Magis 1996.

19 Izazola Licea 1996, Bronfman y Magis 1996, Del Río y Liguori 1995, Weeks 1995, Elias 1991, y Heise y Elias 1995. 
de riesgo (sexo entre varones y uso de drogas intravenosas). En Sudamérica, varios países del sureste asiático, Europa Oriental y África del Norte, la epidemia tuvo patrones mixtos de transmisión, aunque la heterosexual primó en la mayoría de países subdesarrollados ${ }^{20}$. Según el Population Reference Bureau ${ }^{21}$, existieron tres patrones claros: en el África Subsahariana, las mujeres representaban la mayor proporción de muertes; en el sur y sureste asiático, los niños eran los mayormente afectados; y en América Latina y en Norteamérica, los hombres eran las víctimas predominantes.

\section{Aspectos del diagnóstico}

El diagnóstico de seropositivo se relacionó con grupos identificados y señalados por sus prácticas sexuales, en especial con la homosexualidad, la bisexualidad, la prostitución y la promiscuidad, prácticas vedadas y marcadas como degradaciones y perversiones. Al dolor y el temor que la enfermedad que por sí misma producía, se le agregaban la vergüenza y la culpa. Se pensaba que ser diagnosticado como seropositivo implicaba traer a la luz prácticas sexuales ocultas. Además de que frecuentemente se le asociaba con una muerte rápida, se pensaba que el enfermo de sida era víctima de un mal que él mismo propició, del cual era culpable y por lo tanto merecía castigo. Otros consideraban al sida con las más diversas metáforas, como castigo divino, plaga moderna, nuevo holocausto, invento del diablo, genocidio pasivo o fruto del pecado. La Iglesia católica denominó a las personas que tenían esta enfermedad como «los leprosos del siglo XX». Por ello, no en vano el Papa Juan Pablo II propuso a San Luis Gonzaga (modelo de castidad) como santo patrono de los enfermos de sida. Por lo tanto, resultaba muy difícil hablar del sida como problema de salud sin asociarlo a sangre, sexo, muerte y culpabilidad, pues este era un tema que afectaba profundamente la intimidad, la libertad y los valores morales de las personas y de las sociedades ${ }^{22}$.

En tal sentido, en la mayoría de los casos las pruebas se realizaban para conocer si un(a) esposo(a), compañero(a) sexual, madre o padre, familiar, amigo(a) o compañero(a) de trabajo estaba infectado con el virus VIH; la tendencia era apartar y abandonar a las personas que tenían esta enfermedad, sin saber que dichas actitudes no los iban a proteger del contagio y que solo empeorarían más la situación de los enfermos ${ }^{23}$.

20 Potts y May 1987, Piot y Careal 1988, The Johns Hopkins University 1987, Gwede y McDermott 1992, Hospedales 1992, y Onusida y Organización Mundial de la Salud 1998.

21 Carvalho 1992.

22 Izazola Licea 1996, Rubenson 1996, Rodríguez y otros 1992, Connor 1998, Aller Atucha 1988, Carvalho 1992, y Rotheram-Borus, Mahler y Rosario 1995.

23Departament de Sanitat i Seguretat Social 1993, Rubenson 1996, y Galíndez, Milanese y Ternero 1992. 
Según Merson ${ }^{24}$, esto sucedió porque siempre se prefirió negar la conducta sexual cuando implicaba un riesgo para uno mismo y los seres amados.

Este miedo era una reacción tanto en la persona infectada como en los miembros de la comunidad. Ese miedo engendraba, además, indignación con consecuencias nefastas; se dieron casos en los que una persona infectada ha matado a la persona que le contagió el virus o ha decidido infectar a otros para no quedar sola con esta enfermedad ${ }^{25}$.

Por ello, frente a las malas noticias, la primera reacción de la persona afectada era la conmoción, seguida de rechazo e incredulidad. Después, cuando se aceptaba la realidad, se suscitaban intensas reacciones emotivas como el miedo, la indignación y la tristeza. Frente a esa crisis se podía adoptar una actitud positiva tratando de dialogar, de aceptar la situación y de compartir la pena, o una actitud negativa de reproches y aislamiento. Durante este periodo las personas necesitaban mucho apoyo para poder hacer frente a la situación de forma constructiva ${ }^{26}$.

En el inicio de la epidemia, cuando las condiciones de transmisión no estaban aún claramente establecidas, era común ver a los familiares y amigos de los enfermos evitar la cercanía de estos en los servicios de salud (por ejemplo, se quedaban en la puerta del cuarto). Cuando el enfermo dejaba el hospital, lo rechazaban, abandonaban o discriminaban. Muchos profesionales de la salud se rehusaron a prestar asistencia a los casos de sida, porque otros colegas se habían contaminado atendiendo a este tipo de pacientes. Los profesionales de la salud que se dedicaron a atender a las primeras personas con sida se diferenciaron de todos los demás, eran únicos. Algunos tuvieron que optar por cuidar a los pacientes vestidos como «astronautas» (con trajes especiales, cascos, botas, etc.) o, de lo contrario, sin protección, teóricamente exponiéndose, pero preservando el lado humano y personal de la relación médico-paciente ${ }^{27}$.

\section{Aspectos económicos}

El impacto del sida ocurría en esferas inconmensurables de sufrimiento, luto, cambios, pérdidas globales, creatividad interrumpida, generaciones y subculturas aniquiladas. Se podía contar el número de vidas humanas que una sociedad perdía o contabilizar el impacto económico que representaba la pérdida de vidas en edad productiva ${ }^{28}$. Además de la

24Merson 1996.

25 Izazola Licea 1996.

26 Rubenson 1996.

27 Fermina Acuba 1993 y Departamento de Salud y Servicios Humanos 1990.

28 Koop 1987, Organización Panamericana de la Salud 1993 y Fundación para el Estudio e Investigación de la Mujer 1994. 
pérdida afectiva de familiares, las familias también enfrentaban la pérdida de ingresos por desaparición de quienes sostenían el hogar como consecuencia del avance de la enfermedad ${ }^{29}$. También se podía calcular lo que costaba tratar a un enfermo con sida, lo que se gastaba en internarlo en el hospital, medicamentos, personal especializado, exámenes, acompañamiento ambulatorio, alimentación, etc., y, sobre todo, si al paciente le retiraban la cobertura del seguro, podía necesitar ayuda económica especial para obtener el tratamiento adecuado ${ }^{30}$.

El impacto económico de esta enfermedad pudo ser medido mediante los costos directos, como son los costos que se utilizaban para curar pacientes con sida, y los costos indirectos, como eran los ingresos que se perdían al incrementarse la mortalidad en las personas que estaban en edad de trabajar ${ }^{31}$. En lugares de mayor prevalencia del sida, su repercusión en la economía fue considerable. Por ejemplo, en 1991 le costó a las empresas norteamericanas 55 mil millones de dólares en pérdidas de productividad y 8.500 millones en costos de atención en salud ${ }^{32}$.

Según relatos de enfermos con sida, una persona que recurría a la medicina privada podía llegar a gastar 3.600 dólares mensuales apenas en medicamentos cuando tenía un cuadro clínico severo de infecciones oportunistas, sin tener en cuenta el costo de las consultas médicas e internamientos en las clínicas privadas. Para 1992, el costo anual para combatir el sida en Estados Unidos se había incrementado de cinco mil millones a 13 mil millones de dólares. Estos costos no incluían los gastos de drogas antivirales en personas asintomáticas infectadas con el $\mathrm{VIH}^{33}$.

Para la doctora Foster, del London School of Hygiene \& Tropical Medicine, la sociedad en todos sus niveles estaba profundamente afectada por el sida, desde los individuos y sus familiares hasta la economía nacional (en términos de producción de bienes para el consumo interno y el mercado de exportación). Cada muerte por sida acarreaba consigo la pérdida de un promedio de 15 a 20 años de experiencia laboral; se perdió también la inversión en educación y capacitación, junto con la tercera parte del producto que esa persona hubiese podido ganar el resto de su vida. También se estimaba que el VIH causaba la pérdida de 48 días per cápita por año ${ }^{34}$. 


\section{Aspectos educativos}

El problema del sida, además de generar controversias y conflictos sociales, generó angustia, incapacidad y temor en la población, lo cual se debió a las mismas características de la enfermedad (incurable y mortal), así como a las connotaciones que giraban a su alrededor (aspectos sexuales, utilización de drogas, aspectos religiosos, morales, etc.). Por ello se tornó un área muy susceptible a la influencia social y a la fácil distorsión. Esto estaba dado, entre otras cosas, por la poca información que existía, ofrecía y entregaba sobre el sida, la sexualidad y la situación real del problema; la información distorsionada que brindaban los medios masivos de comunicación o algunos grupos e instituciones; las controversias en las opiniones de los grupos en el poder; la rigidez y contradicción de algunas normas y prácticas sociales respecto a la sexualidad; la legalidad; los derechos, etc. ${ }^{35}$.

El éxito o fracaso en el control del sida dependió de la rapidez con que podía frenarse la propagación del VIH mediante una adecuada información pública y de lo que la comunidad médica lograra descubrir tratamientos o medidas preventivas adecuadas. Cada país debió desarrollar estrategias en la prevención de esta enfermedad desde sus valores, cultura, tradiciones, identidad, de acuerdo con sus posibilidades económicas y usando el lenguaje de la población objetivo ${ }^{36}$.

No se trataba solamente de ofrecer información en el sentido estricto de la palabra, sino de crear una conciencia en el público respecto a las dimensiones sociales, psicológicas y afectivas de la enfermedad, y la necesidad que tenía cada persona de responsabilizarse y de implicarse en este asunto; se trataba también de los aspectos éticos y sociales relacionados con el mejoramiento de las condiciones de vida, el respeto de los derechos humanos y la lucha contra la discriminación hacia las personas contaminadas con el virus VIH y los enfermos de sida, ya que esta enfermedad se expandió por la ignorancia. ${ }^{25}$

Asimismo, el conocimiento de la información básica sobre el sida no era suficiente para cambiar los comportamientos. Para que estos se modificaran, los individuos necesitaban entender qué elementos constituían una conducta de alto riesgo, reconocer su riesgo individual y su vulnerabilidad, así como la gravedad de las consecuencias del riesgo relacionado con la conducta sexual. Además de comunicar los beneficios de una conducta de bajo riesgo, los programas de prevención tuvieron que dar a las 
personas los conocimientos y las habilidades necesarias para practicar el sexo más seguro ${ }^{37}$.

Por eso, información, prevención, capacitación y solidaridad con las personas que vivían con el VIH eran los cuatro objetivos principales asignados por los poderes públicos y las asociaciones de lucha contra el sida a las campañas de sensibilización de la opinión pública. Se trataba, en particular, de propiciar la adquisición de comportamientos con riesgo mínimo y esto suponía una evolución compleja en que los aspectos psicológicos y sociales tenían una importancia determinante; por lo tanto, era esencial introducir en este proceso de sensibilización los aspectos culturales propios de cada comunidad, para que los mensajes de prevención y de solidaridad concordaran con la realidad del destinatario ${ }^{38}$.

\section{Conclusiones}

Para conocer lo que estaba sucediendo social y culturalmente con esta epidemia, se necesitaba de más investigaciones desde el punto de vista social. Por ello, las ciencias sociales hicieron notar que los datos cuantitativos no eran suficientes para analizar la incidencia de los diferentes comportamientos sexuales con respecto al VIH y el sida. Es así que a este tipo de investigación se sumó la metodología cualitativa, a fin de tener una mejor comprensión del problema ${ }^{39}$. Aun así, las investigaciones en ciencias sociales demostraron una pasividad ante los retos que demanda la crisis del sida ${ }^{40}$. Para Gogna ${ }^{41}$, este problema tuvo su raíz en que «las ETS no fueron foco de interés de la investigación sociocultural».

Además, la enorme variabilidad del comportamiento heterosexual, con grandes diferencias entre segmentos sociales, culturales, grupos religiosos y países, hizo imposible el establecimiento de un único valor para el riesgo de adquirir la infección. Por ello, los datos mostrados por la literatura eran diferentes según el contexto en el que se realizaron los estudios $^{42}$.

Finalmente, se sabía que la palabra sida significaba síndrome de inmuno deficiencia adquirida; pero dicha enfermedad también tuvo un significado social, mediante el cual se podría resumir lo que la sociedad creía, pensaba y sentía y de cómo trataba a sus enfermos:

37 Carvalho 1992, y Gordon y otros 1988.

38 KIT, Safaids y Organización Mundial de la Salud 1995, y Piot 1996.

39 Yelibi 1993.

40 Lamptey 1995

41 Aggleton 1998.

42 Kallings 1988. 


Sociedad Sentimiento

Intolerante Indiferente

Discrimina y De

Aísla Ayuda

De la misma manera, se esperaba que la definición social del sida, para bien de las personas que tenían esta enfermedad, fuese:

Sociedad

Incorpora

Discriminados a la

Actividad
Sentimiento

Integrado

De

Acción 


\section{REFERENCIAS BIBLIOGRÁFICAS}

AGGLETON, Peter (1997). «What works best». En AIDS Action, nro. 39, p. 2. AGGLETON, Peter y otros (1994). «Risking everything? Risk behavior, behavior care, and AIDS». En Science, nro. 265, pp. 341-345.

ALLER ATUCHA, Luisa (1988). "Aclaraciones necesarias con respecto al sida». En Lugar de Encuentro, vol. 2, nros. 3-4, pp. 1-2.

APPROPIATE HEALTH RESOURCES Y TECHNOLOGIES ACTION GROUP (1996). «One world». En AIDS Action, nro. 34, p. 7.

BASTOS, Cristiana y otros (1994). «Introdução». En Richard Parker y otros (editores). A Aids no Brasil. Brasilia: Relume-Dumará Editores.

BRANDT, Allan (1986). «AIDS: from social history to social policy». En Journal of Law, Medicine and Ethics, vol. 14, nros. 5-6, pp. 231-242.

BRONFMAN, Mario y MAGIS, Carlos (1996). «Tercermundización, despauperización y ruralización». En Demos. Carta Demográfica de México, nro. 9, pp. 42-43.

CÁCERES, Carlos (1995). «La construcción epidemiológica del sida». En Ellen Hardy, Maria Jose Duarte Osis y Evely Rodrigues Crespo (editoras). Ciências Sociais e Medicina. Atualidades e Perspectivas Latino-Americanas. Sâo Paulo: Cemicamp.

CÁCERES, Carlos y ROSASCO, Ana María (1992). «Viviendo positivamente». En Construyendo una vida positiva. Un manual informativo y de orientación para personas con VIH/SIDA y sus familiares y amigos. Lima: Asociación de Lucha contra el Sida Vía Libre.

CARVALHO, João Alberto (1992). «Sexo-pecado, doença/punição: a AIDS entre nós». En Lydia S. Bond (editora). A Portfolio of AIDS/STD Behavioral Interventions and Research. Washington: Organización Panamericana de la Salud.

CHILD, R. (s/f). «VIH: cifras, políticas y algo más». Santiago de Chile: Programa de Prevención de Sida en Chile.

COATES, Thomas (1991). «Principios de la modificación del comportamiento». En Network en Español, vol. 6, nro. 2, pp. 3-5.

CONNOR, Susan (1988). «Sida: aspectos sociales, jurídicos y éticos de las tercera epidemia». En Boletín de la Oficina Sanitaria Panamericana, vol. 105, nros. 5-6, pp. 587-604.

DEPARTAMENT DE SANITAT I SEGURETAT SOCIAL (1993). «Por un futuro sin sida. No te desentiendas. Infórmate. Barcelona: Departament de Sanitat i Seguretat Social. 
DEPARTAMENTO DE SALUD Y SERVICIOS HUMANOS (1990). Consejería y análisis voluntarios para HIV: hechos, temas y respuestas. Atlanta: CDC.

DEPARTMENT OF HEALTH AND HUMAN SERVICES (2000). «Healthy People 2000. Review 1998-1999. HIV infection». En 2000 National Health Promotion and Disease Prevention. Objectives, Priority Area 18, pp. 176-178.

DEL RÍO, Carlos y LIGUORI, Ana Luisa (1995). «Cuestiones de política y control de la infección por VIH». En Enfoques de investigación sobre VIH/SIDA en salud reproductiva, nro. 9, pp. 115-126.

ELIAS, Christopher (1991). "Sexually transmitted diseases and the reproductive health of women in developing countries». En The Population Council, Working Papers, nro. 5.

FAMILY HEALTH INTERNATIONAL (1998). «Caso de SIDA en 1959». En FHI, vol. 18, nro. 3, p. 2.

FERMINA ACUBA, Ajonye (1993). «El sida es una tarea de todos». En Acción en sida, nro. 18, p. 4.

FRANKERBERG, Ronald (1998). "Social and cultural aspects of the prevention of the three epidemics (HIV infection, AIDS and counterproductive societal reaction to them)». En Alan Fleming (editor). The Global Impact of AIDS. Proceeding of the First International Conference on the Global Impact of AIDS. Nueva York: Organización Mundial de la Salud y London School of Hygiene \& Tropical Medicine.

FRASCA, Tim (1993). «La vida es una enfermedad de transmisión sexual». Seminario «Sexualidad, Reproducción y Servicios de Salud», Santiago de Chile.

FUNDACIÓN PARA EL ESTUDIO E INVESTIGACIÓN DE LA MUJER (1994). «Sida y la familia». En Revista DESIDAMOS, vol. 2, nro. 2, p. 18.

GALÍNDEZ, Jorge; MILANESE, Ariel, y TERNERO, María Elena (1992). «El fenómeno sida entre jóvenes de 14 a 24 años en la ciudad de Rosario, Argentina». En Lydia S. Bond (editora). A Portfolio of AIDS/STD. Behavioral Interventions and Research. Washington: Organización Panamericana de la Salud.

GORDON, Gill y otros (1988). «Participatory approaches to the production and use of the mass media». En Alan Fleming (editor). The Global Impact of AIDS. Proceeding of the First International Conference on the Global Impact of AIDS. Nueva York: Organización Mundial de la Salud y London School of Hygiene \& Tropical Medicine

GWEDE, Clement y MCDERMOTT, Robert (1992). «AIDS in Sub-Saharan Africa: implications for health education». En AIDS Education and Prevention, vol. 4, nro. 4, pp. 350-361. 
HEISE, Lori y ELIAS, Christopher (1995). «Transforming AIDS prevention to meet women's needs: a focus on developing countries». En Social Science and Medicine, vol. 40, nro. 7, pp. 933-943.

HOSPEDALES, James y otros (1992). «Epidemiology of HIV/AIDS in the Caribbean». En Peter Lamptey y otros (editores). The Handbook for AIDS. Prevention in the Caribbean. Nueva York: Family Health International.

IZAZOLA LICEA, José Antonio (1996). El impacto y los costos del VIH/SIDA. México D. F.: Iniciativa Regional para la Prevención y Control del sida y otras ETS en América Latina y el Caribe.

KALLINGS, Lars Olof (1988). «Interventions policies». En Alan Fleming (editor). The Global Impact of AIDS. Proceeding of the First International Conference on the Global Impact of AIDS. Nueva York: Organización Mundial de la Salud y London School of Hygiene \& Tropical Medicine.

KELLY, Jeffrey y otros (1992). «Producing population-wide reductions in HIV risk behavoir among small-city gay men». En Lydia S. Bond (editora). A Portfolio of AIDS/STD Behavioral Interventions and Research. Washington: Organización Panamericana de la Salud.

KIT, SAFAIDS y ORGANIZACIÓN MUNDIAL DE LA SALUD (1995). «Can women and men reduce risks and share responsabilities?» En Facing the Challenges of HIV/AIDS/STD: A Gender-Based Response, The Netherlands, pp. 23-42.

KOOP, Everett (1987). Síndrome de inmuno-deficiencia adquirida (AIDS). Informe del jefe del Servicio de Salud Pública de los Estados Unidos. Washington: Servicio de Salud Pública de los Estados Unidos.

LAMPTEY, Peter (1995). «Disminuyendo la transmisión del SIDA: lecciones de una década de esfuerzos y prevención». En AIDS Captions en Español.

LISKIN, Laurie y otros (1989). «Educación sobre el sida: un buen comienzo». En Population Reports, serie L, vol. XVII, nro. 3, p. 8.

MENEGHIN, Paolo; MOURA, Edilene, y LEE, Susana (1995). «AIDS: Uma crise de saúde (e comunicacao) pública». En Fernando Zacarías y Mercedes Weissenbacher (editores). Inventario de recursos de investigación en sida, 1991-1994. América Latina y el Caribe. Washington: Organización Panamericana de la Salud.

MERCER, Hugo (1985). «As contribuiçõos da sociologia a pesquisa em saúde», En Everardo Duarte Nunes (editor). As ciências sociais em saúde na América Latina: tendências e perspectivas. Brasilia: Organización Panamericana de la Salud.

MERSON, Michael (1996). «Reflexiones sobre la respuesta de Estados Unidos a la epidemia de VIH/SIDA». En Revista DESIDAMOS, vol. 4, nros. 3-4, pp. 17-19. 
MONTAGNIER, Luc y otros (1993). Sida. Los hechos, la esperanza. Barcelona: Fundación La Caixa.

ONUSIDA y ORGANIZACIÓN MUNDIAL DE LA SALUD (1998). Informe sobre la epidemia mundial de VIH/SIDA. Ginebra: Organización Mundial de la Salud.

ORGANIZACIÓN PANAMERICANA DE LA SALUD (1993). «Sida. La epidemia de los tiempos modernos». En Comunicación para la Salud, nro. 5.

PAICHELER, Geneviève (1992). «Society facing AIDS». En Michaël Pollak, Geneviève Paicheler y Janine Pierret (editores). AIDS: A Problem for Sociological Research. Londres: Sage Publications.

PARKER, Richard (1993). «Crónica de um debate anunciado». En Boletim Associação Brasileira Interdisciplinar de AIDS, vol. 18, nro. 7, nro. 1-4.

PARKER, Richard y GAGNON, John (editores). Conceiving Sexuality: Approaches to Sex Research in a Postmodern World. Londres y Nueva York: Routledge.

PIOT, Peter (1996). «VIH/SIDA, derechos humanos y responsabilidades humanas». En Revista DESIDAMOS, vol. 4, nros. 3-4, pp. 11-13.

PIOT, Peter y CARAËL, Michael (1988). «Epidemiological and sociological aspects of HIV-infection in developing countries». En British Medical Bulletin, vol. 44, nro. 1, pp. 68-88.

POSADA, J. (1995). «Factores socioculturales que contribuyen a la expansión del VIH/SIDA y su relación con el consumo de drogas». En Fernando Zacarías y Mercedes Weissenbacher (editores). Inventario de recursos de investigación en sida, 1991-1994. América Latina y el Caribe. Washington: Organización Panamericana de la Salud.

POTTS, Malcolm y MAY, R. (1987). Sida: enfrentando la amenaza mundial. Family Health International.

REYES, H. (1993). «El peor de todos los males». En SIDAmérica. Información sobre el sida en las Américas. Washington: Punto de Vista y Panos Institute.

RODRÍGUEZ, Gonzalo y otros (1992). Mitos sobre la sexualidad y el sida en población adolescente. La comunidad de Santa Fe. México D. F.: Mexfam.

ROTHERAM-BORUS, Mary Jane; MAHLER, Karen, y ROSARIO, Margaret (1995). «AIDS prevention with adolescents». En AIDS Education and Prevention, vol. 7 , nro. 4 , pp. 320-336.

RUBENSON, Birgitta (1987). ¿Qué es el sida? Manual para agentes de salud, Comisión Médico Cristiana (CMC). Ginebra: Consejo Mundial de Iglesias.

(1996). Lo que hay que saber sobre el sida. Un manual para pastores y maestros. Ginebra: Acción de las Iglesias por la Salud, Ex Comisión Médica Cristiana. 
THE JOHNS HOPKINS UNIVERSITY (1987). El sida: una crisis de salud pública. En Population Reports, Serie L(6).

(1993). «Control de las enfermedades de transmisión sexual». En Population Reports, serie L, vol. XXI, p. 1.

TREICHELER, Paula (1992). «AIDS, HIV and the cultural construction of reality». En Gilbert H. Herdt, Shirley Lindenbaum (editores). The Time of AIDS. Social Analysis, Theory, and Method. California: Sage Publications.

WEEKS, Jefffry (1995, abril). «Los valores sexuales en la era del sida». En Debate Feminista, nro. 4, p. 157-182.

YELIBI, Sibili (1993). «Sociocultural aspects of AIDS in an urban peripheral area of Abidjan». En AIDS Care, vol. 5, nro. 2, pp. 187-197.

ZALDUONDO, Barbara y BERNARD, Jean M. (1995). «Meanings and consequences of sexual-economic exchange: gender, poverty and sexual risk behavior in urban Haiti». En Richard Parker y John Gagnon. Conceiving Sexuality: Approaches to Sex Research in a Postmodern World. Nueva York: Routledge.

Recibido: Noviembre de 2012.

Aceptado: Febrero de 2012. 